\title{
Comparison of slit mesh versus nonslit mesh in laparoscopic extraperitoneal hernia repair
}

\author{
Dogan Yildirim ${ }^{1}$, Turgut Donmez ${ }^{2}$, Halim Ozcevik ${ }^{3}$, Mikail Cakir $^{1}$, Suleyman Demiryas ${ }^{4}$, Okan Murat Akturk ${ }^{1}$ \\ ${ }^{1}$ Department of General Surgery, Haseki Training and Research Hospital, Istanbul, Turkey \\ ${ }^{2}$ Department of General Surgery, Lutfiye Nuri Burat State Hospital, Istanbul, Turkey \\ ${ }^{3}$ Istanbul Provincial Health Directorate, Istanbul, Turkey \\ ${ }^{4}$ Department of General Surgery, Medicine Faculty, Istanbul Universty Cerrahpasa, Istanbul, Turkey
}

Videosurgery Miniinv 2018; 13 (4): 469-476

DOI: https://doi.org/10.5114/wiitm.2018.77258

\begin{abstract}
Introduction: Endoscopic hernia repair integrates the advantages of tension-free preperitoneal mesh support of the groin with the advantages of minimally invasive surgery procedures.

Aim: To compare outcomes between slit mesh (SM) and nonslit mesh (NSM) placement in laparoscopic totally extraperitoneal (TEP) inguinal hernia repair.

Material and methods: This is a retrospective study of 353 patients who underwent TEP inguinal hernia repair between January 2010 and December 2011. One hundred forty-nine and 154 hernias were operated on in the SM and NSM groups, respectively. Postoperative complications, recurrence, early postoperative pain, and chronic pain levels were evaluated.

Results: In a total of 303 patients, hernia repair was performed as 395 direct and indirect hernias. Nonslit mesh was converted from TEP to transabdominal preperitoneal patch plasty (TAPP) in 4 patients in the group and 6 patients in the slit mesh group. The average operation time of the SM group was significantly higher than that of the NSM group $(p<0.001)$. In the evaluation of early postoperative pain, VAS levels of the NSM group were statistically significantly lower than those of the $S R$ group in all evaluations $(p=0.001)$. The pain rate of the $S M$ group after 3 months of chronic pain was significantly higher than that of the NSM group $(p=0.004)$. There was no difference in recurrence rate, $6^{\text {th }}$ month chronic pain, wound infection or wound hematoma.

Conclusions: The use of SM and NSM in TEP operations is not different in terms of recurrence and complications. However, the use of NSM gives better results in terms of early postoperative pain and chronic pain.
\end{abstract}

Key words: total extraperitoneal, groin hernia, slit mesh, nonslit mesh.

\section{Introduction}

Inguinal hernia repairs are among the most widely performed operations. Annually, 700,000 hernia operations are carried out in the USA [1]. Several inguinal hernia procedures have been described, including McVay, Bassini, posterior wall repair, and anterior and posterior repairs with synthetic mesh.
Lichtenstein operations are considered the gold standard and reportedly reduce the rate of recurrence $[2,3]$. Endoscopic procedures have been performed for at least a few decades now. Laparoscopic total extraperitoneal repair (TEP) is considered to be advantageous compared to other procedures, resulting in reduced postoperative and chronic pain and better cosmetic results. Unlike other endoscop-

Address for correspondence

Turgut Donmez MD, Department of General Surgery, Lutfiye Nuri Burat State Hospital, Bakırkoy Dr. Sadi Konuk Training and Research

Hospital, Zuhuratbaba Mahallesi, Akıl Hastanesi Cd No:11, Bakırkoy, 34147 Istanbul, Turkey, phone: +90 5347400967,

e-mail: surgeont73@hotmail.com 
ic procedures, TEP prevents intraperitoneal access, thus decreasing the development of peritoneal complications, such as intestinal or colonic damage or postoperative adhesions [4-6]. The recurrence rates for TEP have been reported as $1-4 \%$. The principal disadvantage of TEP is its steep learning curve, with results being related to the experience of the surgeon [1].

The recurrence rate following TEP repair is between $1 \%$ and $10 \%[7,8]$. Making a slit in the mesh allows it to be positioned behind the cord structures, which might be a risk factor for recurrence [9]. One study has suggested that some recurrences are associated with insufficient closure of the mesh slit [10]. The Current International Endohernia Society Guidelines point out that recurrences might arise from insufficient measures of the mesh, an overly large diameter of the hernia sac, and a slitted mesh [9].

However, there are conflicting reports about the rates of recurrence when using slit (SM) and nonslit mesh (NSM) [9]. Also, testicular pain is reported to be higher in TEP operations with SM compared with NSM operations [11].

\section{Aim}

Here we aimed to determine whether there is a difference between the slit and the nonslit approaches. We compared the two techniques regarding recurrence rate, postoperative complications, and effect on the patient's life.

\section{Material and methods}

The data of patients who underwent laparoscopic inguinal hernia repair at the Department of Surgery (Haseki Training and Research Hospital and Lutfiye Nuri Burat State Hospital), between May 2014 and May 2017, were collected from the computerized hospital database. We retrospectively analyzed the 353 patients with inguinal hernia who underwent TEP. A review of patient's charts and a follow-up questionnaire were performed by phone in December 2017 to all available patients. Finally, thorough physical examinations and ultrasonography were done for evaluation of hernia recurrence.

Recurrent, strangulated or incarcerated hernias, bilateral inguinal hernias, and patients with co-morbid diseases (e.g., rheumatoid arthritis, severe hypertension, severe heart insufficiency, bleeding diathesis, joint disease, hip and knee prostheses, and neurologic sequelae) were excluded. All the patients were informed of the surgical technique and its possible complications by the responsible surgeon. Written informed consent was taken from all the patients. Laparoscopic operations were performed by two well-experienced surgeons and their team. Three hundred fifty-three patients who were followed up for 1 year were divided into two groups: slit mesh TEP (group I, $n=149$ patients) and nonslit mesh TEP (group II, $n=154$ patients). Demographic characteristics, postoperative pain, hospital stay and return to work period, early and late complications, recurrence, and chronic pain scores were recorded. Pain scoring was done using a visual analog scale (VAS) (zero = no pain; 10 = intractable pain).

A patient satisfaction survey was done using a 5-parameter Likert scale. The patients were asked their satisfaction with the surgery during the third month of follow-up. Pain, healing, and return to daily activity were questioned. Points were 1 for very dissatisfied; 2 for dissatisfied; 3 for neutral; 4 for satisfied; and 5 for very satisfied. The local ethical committee approved this study (Haseki Ethical Committee no: R6 date: 20/02/2018 and IRB number: 37 date: 15/02/2018).

\section{Surgical methods}

The TEP procedure was the preferred approach. TEP was performed using a $10-\mathrm{mm}$ balloon placemaker inserted through a right periumbilical incision and replaced by a blunt trocar. A 10-mm, 30-degree camera was used routinely in all cases. $\mathrm{CO}_{2}$ insufflation of the space of $10-12 \mathrm{~mm} \mathrm{Hg}$ pressure was provided. A median $5 \mathrm{~mm}$ trocar was inserted at the symphysis pubis under direct vision. Another median $5 \mathrm{~mm}$ trocar was also inserted at the midpoint between these two trocars. Dissection of the chamber was performed to visualize inferior epigastric vessels, inferior parts of the rectus muscle, and the symphysis pubis. The Cooper ligament was dissected to the point where it met the femoral vein, and the iliopubic tract was exposed. The spermatic cord was found, and the hernia sac was separated from the cord and reduced. A $100 \times 150 \mathrm{~mm}$ mesh was used to cover the myopectineal orifice, Hasselbach area, and femoral canal orifice, and fixed to the symphysis pubis with an absorbable tacker. (According to the EHS classification, $120 \times 170 \mathrm{~mm}$ mesh was placed in M3 hernias.) NSM included the mesh placed 
above the spermatic cord. During the slit mesh, the lateral edges were cut to form a hole in the middle of the mesh, wrapped behind and around the cord, and the cord passed through the hole in the mesh. There was no difference in preperitoneal dissection between the two groups.

\section{Statistical analysis}

SPSS 15.0 was used for statistical analysis. For descriptive statistics, categorical parameters were given in numbers and percentages, and numeric parameters were presented as mean, median, standard deviation, minimum, and maximum. When parametric requirements were met, two independent groups were compared using Student's t-test. Otherwise the Mann-Whitney $U$ test was used. Rates were compared using the $\chi^{2}$ test and with Monte Carlo simulation (when needed). The significance level was $p<0.05$

\section{Results}

Between May 2014 and May 2017, 303 patients (283 male and 20 female) underwent laparoscopic TEP hernia repair (a total 395 hernia repairs). One hundred forty-nine patients had SM, and 154 patients had NSM (Table I). Four patients in the nonslit group and 6 patients in the SM hernia repair group converted to TAPP (Table II). In the follow-up, the patients were phoned to check in for an examination about hernia recurrence. Two hundred ninety-four patients were manually examined for a hernia recurrence. One hundred forty-three patients in the 149-patient SM group, and 151 patients in the 154-patient NSM group were examined manually for a recurrence ( $96 \%$ and $98 \%$, respectively).

The median follow-up was 26 months (range: 16-56) for the SM group and 26 months (range: 1634) for the NSM group (Table II). All of the patients were noted for complications. No patients were lost to follow-up. There was no significant difference between the groups in terms of age, sex, body mass index, and ASA scores (Table I). The operation time for the SM group was significantly longer than for the NSM group; $p<0.001$. The complications are summarized in Table II. Comparing the two groups, we detected no difference in the recurrence rate, chronic pain in the sixth month, the rate of infection or rate of hematoma.

After 3 months, group 1 had significantly higher chronic pain when compared to group 2. There was no significant difference between the groups' in-hospital stay, time to return to work, and average follow-up period ( $p=0.998, p=0.455$ ). There was no significant difference between the groups in hernia diameters, the incidence of a hernia larger than $5 \mathrm{~cm}$ in diameter, conversion rate, use of drains, recurrence, seroma, scrotal edema, wound infection,

Table I. Demographic data of patients

\begin{tabular}{|c|c|c|c|c|c|}
\hline \multirow[t]{2}{*}{ Parameter } & \multicolumn{2}{|c|}{ Group 1} & \multicolumn{2}{|c|}{ Group 2} & \multirow[t]{2}{*}{$P$-value } \\
\hline & Mean \pm SD & $\begin{array}{l}\text { Min.-max. } \\
\text { (median) }\end{array}$ & Mean \pm SD & $\begin{array}{l}\text { Min.-max. } \\
\text { (median) }\end{array}$ & \\
\hline Age [years] & $38.06 \pm 13.1$ & 18-68 (38) & $37.4 \pm 12.5$ & $18-67(37)$ & 0.456 \\
\hline BMI [kg/m²] & $25.2 \pm 3.1$ & $19.7-33.0(24.8)$ & $24.9 \pm 2.5$ & $15.7-30.9(25)$ & 0.370 \\
\hline Operation duration [min] & $50.4 \pm 7.1$ & $35-75(48)$ & $46.3 \pm 6.1$ & $32-63(46)$ & $<0.001$ \\
\hline Parameter & $n$ & $\%$ & $n$ & $\%$ & $P$-value \\
\hline \multicolumn{6}{|l|}{ Gender: } \\
\hline Female & 13 & 8.7 & 16 & 10.4 & 0.622 \\
\hline Male & 136 & 91.3 & 138 & 89.6 & \\
\hline \multicolumn{6}{|l|}{ ASA: } \\
\hline 1 & 133 & 89.3 & 132 & 85.7 & 0.351 \\
\hline 2 & 16 & 10.7 & 22 & 14.3 & \\
\hline \multicolumn{6}{|l|}{ Hernia type: } \\
\hline Indirect & 116 & & 134 & & 0.465 \\
\hline Direct & 71 & & 74 & & \\
\hline
\end{tabular}


Table II. Operation data

\begin{tabular}{|c|c|c|c|c|c|}
\hline \multirow[t]{2}{*}{ Parameter } & \multicolumn{2}{|c|}{ Group 1} & \multicolumn{2}{|c|}{ Group 2} & \multirow[t]{2}{*}{$P$-value } \\
\hline & Mean $\pm S D$ & $\begin{array}{l}\text { Min.-max. } \\
\text { (median) }\end{array}$ & Mean \pm SD & $\begin{array}{l}\text { Min.-max. } \\
\text { (median) }\end{array}$ & \\
\hline Return to work [days] & $18.95 \pm 3.04$ & $14-26(19)$ & $19.01 \pm 2.53$ & $14-24(19)$ & 0.752 \\
\hline Hospital stay [h] & $29.0 \pm 6.4$ & $21-43(26)$ & $27.1 \pm 2.8$ & $21-38(27)$ & 0.998 \\
\hline Duration of follow-up [months] & $26.1 \pm 5.8$ & $16-56(26)$ & $25.2 \pm 4.8$ & $16-34(26)$ & 0.455 \\
\hline Parameter & $n$ & $\%$ & $n$ & $\%$ & $P$-value \\
\hline \multicolumn{6}{|l|}{ Hernia diameter $[\mathrm{cm}]:$} \\
\hline$<5$ & 142 & 95.3 & 149 & 96.8 & 0.517 \\
\hline$\geq 5$ & 7 & 4.7 & 5 & 3.2 & \\
\hline Conversion & 6 & 4.0 & 4 & 2.6 & 0.536 \\
\hline Drain & 8 & 5.4 & 11 & 7.1 & 0.524 \\
\hline Recurrence & 6 & 4 & 5 & 3.2 & 0.717 \\
\hline Seroma & 15 & 10.1 & 10 & 6.5 & 0.258 \\
\hline Scrotal edema & 14 & 9.4 & 9 & 5.8 & 0.243 \\
\hline Surgical site infection & 6 & 4.0 & 5 & 3.2 & 0.717 \\
\hline Post-operative pain at 3 months & 16 & 10.7 & 4 & 2.6 & 0.004 \\
\hline Post-operative pain at 6 months & 4 & 2.7 & 1 & 0.6 & 0.208 \\
\hline
\end{tabular}

or pain after 6 months (Table II). In the early postoperative period, in pain evaluation, group 1 had significantly lower visual pain score levels in all evaluations compared to group 2 (Table III).

When hernia diameters were evaluated in detail, there was no difference between the two groups, nor any difference between hospital stay and complication rate. In the group with hernia diameter less than $5 \mathrm{~cm}$, there was more chronic pain in the SM group than in the NSM group ( $p=0.007)$ (Table IV).

In the group with a hernia diameter less than $5 \mathrm{~cm}$, the VPS levels of group 1 were lower in all evaluations when compared to group 2. When the hernia diameter was larger than $5 \mathrm{~cm}$, there was no statistically significant difference in VPS levels between the two groups (Table V).

\section{Discussion}

Several procedures have been used for laparoscopic hernia repair operations, of which TEP and TAPP are commonly used. Total extraperitoneal has an advantage of being extraperitoneal, therefore sparing the intraperitoneal organs from damage. By avoiding large incisions, a laparoscopic hernia combines the advantages of posterior prosthetic mesh hernia repair with the rapid recovery advantage of laparoscopic operations. Laparoscopic TEP

Table III. VAS score (visual analogue scale)

\begin{tabular}{|c|c|c|c|c|c|}
\hline \multirow[t]{2}{*}{ Postop. } & \multicolumn{2}{|c|}{ Group 2} & \multicolumn{2}{|c|}{ Group 1} & \multirow[t]{2}{*}{$P$-value } \\
\hline & Mean \pm SD & Min.-max. (median) & Mean \pm SD & Min.-max. (median) & \\
\hline 1 & $5.11 \pm 0.83$ & $3-6(5)$ & $5.42 \pm 0.68$ & $3-6(6)$ & 0.001 \\
\hline 4 & $2.43 \pm 0.51$ & $2-4(2)$ & $2.92 \pm 0.63$ & $2-4(3)$ & $<0.001$ \\
\hline 12 & $1.34 \pm 0.47$ & $1-2(1)$ & $1.61 \pm 0.56$ & $1-3(2)$ & $<0.001$ \\
\hline 24 & $0.68 \pm 0.51$ & $0-2(1)$ & $0.84 \pm 0.49$ & $0-2(1)$ & 0.004 \\
\hline
\end{tabular}


Table IV. Operation data according to hernia size

\begin{tabular}{|c|c|c|c|c|c|c|c|c|c|c|}
\hline \multirow[t]{2}{*}{ Parameter } & \multicolumn{5}{|c|}{ Hernia diameter $<5$} & \multicolumn{5}{|c|}{ Hernia diameter $\geq 5$} \\
\hline & \multicolumn{2}{|c|}{ Group 1} & \multicolumn{2}{|c|}{ Group 2} & $P$-value & \multicolumn{2}{|c|}{ Group 1} & \multicolumn{2}{|c|}{ Group 2} & $P$-value \\
\hline Hospital stay [h] & \multicolumn{2}{|c|}{$28.8 \pm 6.3$} & \multicolumn{2}{|c|}{$27.1 \pm 2.8$} & 0.904 & \multicolumn{2}{|c|}{$32.4 \pm 8.7$} & \multicolumn{2}{|c|}{$28.0 \pm 2.9$} & 0.625 \\
\hline Duration of follow-up [months] & \multicolumn{2}{|c|}{$25.9 \pm 5.8$} & \multicolumn{2}{|c|}{$25.1 \pm 4.9$} & 0.658 & \multicolumn{2}{|c|}{$30.0 \pm 4.9$} & \multicolumn{2}{|c|}{$25.8 \pm 1.9$} & 0.086 \\
\hline Parameter & $n$ & $\%$ & $n$ & $\%$ & $P$-value & $n$ & $\%$ & $n$ & $\%$ & $P$-value \\
\hline Conversion & 5 & 3.5 & 4 & 2.7 & 0.745 & 1 & 14.3 & 0 & 0.0 & 1.000 \\
\hline Recurrence & 6 & 4.2 & 4 & 2.7 & 0.533 & 0 & 85.7 & 1 & 20.0 & 1.000 \\
\hline Seroma & 10 & 7.04 & 6 & 4.02 & 0.329 & 5 & 71.42 & 4 & 80 & 0.856 \\
\hline Scrotal edema & 10 & 7.04 & 5 & 3.35 & 0.148 & 4 & 57.14 & 4 & 80 & 1.000 \\
\hline Surgical site infection & 5 & 3.5 & 5 & 3.4 & 1.000 & 1 & 14.3 & 0 & 0.0 & 1.000 \\
\hline Post-operative pain at 3 months & 15 & 10.6 & 4 & 2.7 & 0.007 & 1 & 14.3 & 0 & 0.0 & 1.000 \\
\hline Post-operative pain at 6 months & 4 & 2.8 & 1 & 0.7 & 0.205 & 0 & 0.0 & 0 & 0.0 & - \\
\hline
\end{tabular}

Table V. VAS score according to hernia size

\begin{tabular}{|lcccccc|}
\hline Postop. & \multicolumn{3}{c}{ Hernia diameter $<5$} & \multicolumn{3}{c|}{ Hernia diameter $\geq 5$} \\
\cline { 2 - 7 } & Group 2 & Group 1 & $P$-value & Group 2 & Group 1 & $P$-value \\
\hline 1 & $5.11 \pm 0.83$ & $5.42 \pm 0.68$ & 0.001 & $5.29 \pm 0.95$ & $5.40 \pm 0.89$ & 0.855 \\
\hline 4 & $2.42 \pm 0.51$ & $2.90 \pm 0.62$ & $<0.001$ & $2.71 \pm 0.49$ & $3.40 \pm 0.55$ & 0.052 \\
\hline 12 & $1.34 \pm 0.47$ & $1.62 \pm 0.56$ & $<0.001$ & $1.29 \pm 0.49$ & $1.40 \pm 0.55$ & 0.692 \\
\hline 24 & $0.67 \pm 0.50$ & $0.85 \pm 0.49$ & 0.003 & $0.86 \pm 0.69$ & $0.80 \pm 0.45$ & 0.922 \\
\hline
\end{tabular}

is the principally used procedure of laparoscopic hernia repair. The size and type of mesh, the fixation method, and the surgical technique affect the recurrence rates [11]. Fitzgibbons et al. described the reasons for recurrence as follows: the inexperience of the surgeon, inadequate dissection, insufficient mesh size, ineffective coverage of mesh of the hernia defect, failure in fixation, and hematoma formation [12].

The dominant factors in successful preperitoneal hernia repair are an adequate dissection of the space, sufficient exposure, the complete closure of the myopectineal orifice, and satisfactory description of the anatomy. Neumayer et al. compared the open procedure with laparoscopic repair in a randomized clinical trial of 1983 patients and found that the recurrence rate was $10.1 \%$, which was much higher than the open procedure (4.9\%). In this study, 989 patients underwent laparoscopic hernia repair, in $90 \%$ of cases TEP [4]. In another randomized clini- cal study by the MRC group, the recurrence rate was lower (but still high) in the laparoscopic group (1.9\% vs. $0 \%, p=0.017$ ) [13]. McCormick et al. published a meta-analysis of 7161 patients of 41 studies, in which the recurrence rate was lower in the laparoscopic group, but this difference was not statistically significant $(2.74 \%$ vs. $3.11 \%, p=0.16)$ [5].

Slitting the mesh might affect recurrence, as is the case in open surgery. Korman et al. compared different mesh configurations in the laparoscopic TEP procedure of inguinal hernia repair; this randomized clinical trial found no difference in the recurrence rates between the SM and NSM groups [14].

These authors reported recurrence rates of 0.7$2.1 \%$ across the included studies, which supports the use of the SM for TEP operations [15-17]. Domniz et al. compared the SM and NSM mesh in a group of 387 inguinal hernia patients treated with TEP and found lower recurrence rates in the SM group than the NSM group (0.6\% vs. 5.9\%, $p=0.001)$ [18]. 
The supporters of SM argue that this technique has some advantages.

The SM provides an additional fixation point around the spermatic cord structures and prevents the leverage of the mesh from the retroperitoneum by the cord structures, and thus reduces the risk of recurrence. This repair is more anatomical and might be compared to the Lichtenstein procedure in which the cord structures pass through a slit in the mesh. However, there are some problems with this technique. The slit in the mesh around the spermatic cord might be too tight or too loose. The International Endohernia Society released a guideline in 2015 stating that there was no convincing evidence to support the use of a slit or to use no slit in the mesh for laparoscopic inguinal hernia repair. It is not routinely advised to cut a slit in the mesh since it does not bring any technical advantage for the surgeon or better clinical results for the patient $[9,19]$. In our study, the recurrence rate in the SM group was $4 \%$ (6 patients) vs. 3.2\% (5 patients) in the NSM group. There was no significant difference in recurrence between the two groups ( $p=0.717)$.

The size of the mesh may have a bigger effect on recurrence than the surgical technique. Although there is no randomized study comparing different mesh sizes, the diameter of the mesh used in various studies comparing surgery varies. In a meta-analysis of 41 studies of 7446 patients on open and laparoscopic hernia repair procedures, it was found that recurrence rates dropped as the mesh size increased. The use of a small-sized mesh almost doubled the recurrence rate [20].

There have been two large randomized studies conducted in Sweden on endoscopic repair or inguinal hernias. These studies compared TAPP results of 920 patients with Shouldice repair in a 5-year follow-up and reported a recurrence rate of $6.6 \%$ in TAPP with a $7 \times 12 \mathrm{~cm}$ mesh [21]. In another study, the authors compared TEP with the Lichtenstein method in a clinical trial of 1370 patients with a $12 \times 15 \mathrm{~cm}$ mesh, reporting a recurrence rate of 3-5\% [22].

In a guideline released in 2011, the EHS recommends a $10 \times 15 \mathrm{~cm}$ mesh in grade $A$ hernias, and a $12 \times 17 \mathrm{~cm}$ or larger mesh for grade $D$ hernias (direct $>3.4 \mathrm{~cm}$, indirect $>4-5 \mathrm{~cm}$ ) [9]. In our study, for hernias below $3 \mathrm{~cm}$ (direct or indirect), a $10 \times 15 \mathrm{~cm}$ mesh was used, and a $15 \times 20 \mathrm{~cm}$ mesh for hernias larger than $3 \mathrm{~cm}$. It was reported at an EHS congress in Vienna in 2017 that, when the hernia diameter is larger than $5 \mathrm{~cm}$, the recurrence rate increases. In our study, the recurrence rates were higher in the group with a hernia diameter less than $5 \mathrm{~cm}$. This may be because of the use of a larger mesh for hernias with a diameter equal to or larger than $5 \mathrm{~cm}$. There was no difference between the SM and NSM groups.

The fixation technique of the mesh might also play a role in recurrence rates. In 2011, the EHS guideline reported a comparison of two procedures for hernia recurrence, fibrin glue and stapler fixation, with recurrence rates of $0.6 \%$ and $0.4 \%$ respectively, with no significant difference between the two techniques [9]. In our study, absorbable mesh fixation was used in all procedures.

The International Association for the Study of Pain (IASP) describes chronic pain as pain that lasts for more than 3 months, and surgical inventions can often result in chronic pain [23]. In a meta-analysis, Aasvang et al. reviewed 35 clinical studies and reported chronic pain rates of $18 \%$ (range: 0-75\%) and 6\% (range: $1-16 \%$ ) after open hernia repair and laparoscopic hernia repair respectively $(p<0.001)$ [24]. Overall, when the rate of chronic pain is $0-53 \%$, moderate or high pain is seen in $10-12 \%$ of these patients. The use of a mesh has been shown to reduce chronic pain [24]. After hernia repair with a mesh, $11 \%$ of patients suffer from chronic pain, and a quarter of these patients report medium or high levels of pain [24].

Some studies report a significant association of early high postoperative pain after inguinal hernia repair with chronic pain. In the clinical prospective study of Mitura et al., it was emphasized that preoperative evaluation of pain in an inguinal hernia operation has positive effects on postoperative pain, especially in young patients [25]. A few studies have evaluated the occurrence of severe acute pain after endoscopic hernia repair and chronic pain. Two clinical studies, with 313 and 123 patients, reported early postoperative high pain level as a risk factor for chronic pain $(p<0.05$ and $p<0.03$, respectively) $[26,27]$. Ersin et al. reported reduced testicular blood supply in the early postoperative period after TEP hernia repairs, which might contribute to early postoperative pain; however, these authors did not give a specific explanation for chronic long-term pain [28].

Domniz et al. made a retrospective clinical study to investigate chronic pain in SM and NSM groups 
and found no significant difference [18]. In our study, we evaluated the early postoperative pain for patients with a hernia diameter less than $5 \mathrm{~cm}$. For these patients, the postoperative pain level reflected by the VPS was higher in group 1 than group 2, which was statistically significant in the first, $6^{\text {th }}, 12^{\text {th }}$, and $24^{\text {th }}$ hours postoperatively $(p<0.001, p<0.001$, $p<0.001$, and $p=0.004)$. When the chronic pain was investigated at the $3^{\text {rd }}$ month, 16 patients in group 1 (10.7\%) and 4 patients in group 2 suffered from chronic pain, and there was a significant difference between the groups $(p=0.004)$. This is in agreement with other studies. At the $6^{\text {th }}$ month after surgery, there was also a significant difference between group 1 and group 2, with 4 (2.7\%) patients and $1(0.6 \%)$ patient suffering from chronic pain, respectively. When the hernia diameter was larger than $5 \mathrm{~cm}$, there was no significant difference between the groups in the early postoperative period of pain.

Scrotal edema is a common complication after laparoscopic TEP hernia repair. The scrotal edema incidence rate has been reported as $17.8 \%$ in TEP. Lau and Lee, in a clinical study, identified age, large hernia defect, descent of a hernia to the scrotum, and distal remnant hernia sac, as factors related to the formation of scrotal edema [29]. Krishna et al., in a prospective randomized trial, reported a scrotal edema incidence of $9.4 \%$ [30]. To our knowledge, there is no other study which compares slit mesh with nonslit mesh in scrotal edema. Here, 14 (9.4\%) patients in the SM group and 9 (5.8\%) patients in the NSM group had scrotal edema. Overall, the scrotal edema rate was $6.51 \%$ (23 patients). There was no significant difference between the two groups regarding scrotal edema ( $p=0.243)$.

Another common complication of laparoscopic TEP hernia repair is the formation of a seroma. Because a seroma mimics a hernia recurrence, it causes anxiety among the patients. Lau and Lee found that patient age, a large hernia defect, scrotal hernia, and distal indirect hernia sac increase the risk of both scrotal edema and seroma [29]. These authors report a seroma rate of $7.2 \%$ in a study of 450 patients. The incidence of seroma varies between $1.9 \%$ and 28\% [29-31]. Krishna et al., in a randomized study of 100 patients, reported seroma incidence as $28 \%$ in the first week, and about $5 \%$ at the end of the first month [30]. To our knowledge, no study has compared the seroma incidence between slit and nonslit mesh TEP hernia repair. In our study, $25(7.8 \%)$ patients had a seroma - 15 (10.1\%) patients in the SM group, and 10 (6.5\%) patients in the NSM group. There was no difference between the two groups in the rate of seroma formation ( $p=0.258)$. In our study, scrotal edema and seroma formation were more often associated with hernias larger than $5 \mathrm{~cm}$ and scrotal hernias, but this did not reach statistical significance.

\section{Conclusions}

We detected no difference between recurrence rates among the SM and NSM groups after laparoscopic TEP hernia repair. There was no significant difference in scrotal edema and seroma between the two groups. For chronic pain, we detected no significant difference between the two groups at the 6-month follow-up; although there was more chronic pain in the SM group than the NSM group at the 3-month follow-up. When the hernia diameter is equal to or larger than $5 \mathrm{~cm}$, a larger mesh prevents hernia recurrence. Both procedures are safe, efficient, and have low recurrence rates, with high patient satisfaction.

\section{Conflict of interest}

The authors declare no conflict of interest.

\section{References}

1. Belyansky I, Tsirline VB, Klima DA, et al. Prospective, comparative study of postoperative quality of life in TEP, TAPP, and modified Lichtenstein repairs. Ann Surg 2011; 254: 709-14.

2. Mitura K, Romanczuk M. Redundant modifications of Lichtenstein technique in hernia repair - a descriptive study of practicing surgeons in Poland. Videosurgery Miniinv 2009; 4: 1-5.

3. Scott NW, McCormack K, Graham P, et al. Open mesh versus non-mesh for repair of femoral and inguinal hernia. Cochrane Database Syst Rev 2002; 4: CD002197.

4. Neumayer L, Giobbie-Hurder A, Jonasson O, et al. Open mesh versus laparoscopic mesh repair of inguinal hernias. N Eng J Med 2004; 350: 1819-27.

5. McCormick K, Scott NW, Go PM, et al.; EU Hernia Trialists Collaboration. Laparoscopic technique versus open technique for inguinal hernia repair. Cochrane Database Syst Rev 2003; 1: CD001785.

6. Swanstrom L. Laparoscopic surgery: laparoscopic herniorrhaphy. Surg Clinics North Am 1996; 73: 483-91.

7. Staarink M, van Veen RN, Hop WC, Weidema WF. A 10-year follow-up study on endoscopic total extraperitoneal repair of primary and recurrent inguinal hernia. Surg Endosc 2008; 22: 1803-6. 
8. Arregui ME, Young SB. Groin hernia repair by laparoscopic techniques: current status and controversies. World J Surg 2005. 29: 1052-7.

9. Bittner R, Arregui ME, Bisgaard T, et al. Guidelines for laparoscopic (TAPP) and endoscopic (TEP) treatment of hernia [International Endohernia Society (IEHS)]. Surg Endosc 2011; 25: 2773-843.

10. Leibl BJ, Schmedt CG, Schwarz J, et al. A single institution's experience with transperitoneal laparoscopic hernia repair. Am J Surg 1998; 175: 446-52.

11. Chia CL, Su J, Hoe Y, et al. Outcomes of slit mesh in laparoscopic totally extraperitoneal inguinal hernia repair: does it affect recurrence? Asian J Endosc Surg 2015; 8: 434-8.

12. Fitzgibbons RJ Jr, Puri V. Laparoscopic inguinal hernia repair. Am Surg 2006; 72: 197-206.

13. [No authors listed] Laparoscopic versus open repair of groin hernia: a randomized comparison. The MRC Laparoscopic Groin Hernia Trial Group. Lancet 1999; 354: 185-90.

14. Korman JE, Hiatt JR, Feldmar D, Phillips EH. Mesh configurations in laparoscopic extraperitoneal herniorrhaphy. A comparison of techniques. Surg Endosc 1997; 11: 1102-5.

15. Messenger DE, Aroori S, Vipond MN. Five-year prospective follow-up of 430 laparoscopic totally extraperitoneal inguinal hernia repairs in 275 patients. Ann R Coll Surg Engl 2010; 92: 201-5.

16. Napier T, Olson JT, Windmiller J, Treat J. A long-term follow-up of a single rural surgeon's experience with laparoscopic inguinal hernia repair. WMJ 2008; 107: 136-9.

17. Weyhe D, Meurer K, Belyaev O, et al. Do various mesh placement techniques affect the outcome in totally extraperitoneal hernia repair? What is the role of the surgeon? J Laparoendosc Adv Surg Tech 2007; A17: 749-57.

18. Domniz N, Perry ZH, Lantsberg L, et al. Slit versus non-slit mesh placement in total extraperitoneal inguinal hernia repair. World J Surg 2011; 35: 2382-6.

19. Bittner R, Montgomery MA, Arregui E, et al. Update of guidelines on laparoscopic (TAPP) and endoscopic (TEP) treatment of inguinal hernia (International Endohernia Society). Surg Endosc 2015; 29: 289-321.

20. Stengel D, Bauwens K, Ekkernkamp A. Recurrence risks in randomized trials of laparoscopic versus open inguinal hernia repair: to pool or not to pool (this is not the question). Langenbecks Arch Surg 2004; 389: 492-8.

21. Arvidsson D, Berndsen FH, Larsson LG, et al. Randomized clinical trial comparing 5-year recurrence rate after laparoscopic versus shouldice repair of primary inguinal hernia. Br J Surg 2005; 92: 1085-91.

22. Eklund AS, Montgomery A, Rasmussen C, et al. Low recurrence rate after laparoscopic (TEP) and open (Lichtenstein) inguinal hernia repair. A randomized, multicenter trial with 5-year follow-up. Ann Surg 2009; 249: 33-8

23. International Association for the Study of Pain. Classification of chronic pain. Descriptions of chronic pain syndromes and definitions of pain terms. Prepared by the International Association for the Study of Pain, Subcommittee on Taxonomy. Pain Suppl 1986; 3: S1-226.

24. Aasvang E, Kehlet $\mathrm{H}$. Chronic postoperative pain: the case of inguinal herniorrhaphy. Br J Anaesth 2005; 95: 69-76.
25. Mitura K, Śmietański M, Kozieł S, et al. Factors influencing inguinal hernia symptoms and preoperative evaluation of symptoms by patients: results of a prospective study including 1647 patients. Hernia 2018 Apr 26. doi: 10.1007/s10029-0181774-4 (Epub ahead of print).

26. Berndsen FH, Petersson U, Arvidsson D, et al. Discomfort five years after laparoscopic and shouldice inguinal hernia repair: a randomised trial with 867 patients. A report from the SMIL study group. Hernia 2008; 11: 307-13.

27. Poobalan AS, Bruce J, Smith WC, et al. A review of chronic pain after inguinal herniorrhaphy. Clin J Pain 2003; 19: 48-54.

28. Ersin S, Aydin U, Makay O, et al. Is testicular perfusion influenced during laparoscopic inguinal hernia surgery? Surg Endosc 2006; 20: 685-9.

29. Lau H, Lee F. Seroma following endoscopic extraperitoneal inguinal hernioplasty. Surg Endosc 2003; 17: 1773-7.

30. Krishna A, Misra MC, Bansal VK, et al. Laparoscopic inguinal hernia repair: transabdominal preperitoneal (TAPP) versus totally extraperitoneal (TEP) approach: a prospective randomized controlled trial. Surg Endosc 2012; 26: 639-49.

31. Lepere $M$, Benchetrit S, Debaert $M$, et al. A multicentric comparison of transabdominal versus totally extraperitoneal laparoscopic hernia repair using PARIETEX meshes. JSLS 2000; 4: 147-53.

Received: 5.05.2018, accepted: 18.06.2018. 